\title{
Design Considerations of Ultra-Low-Power Polymer Gas Microsensors Based on Noise Analysis †
}

\author{
Rafael Puyol 1, Sylvain Pétré ${ }^{1}$, Yann Danlée ${ }^{1,2}$, Thomas Walewyns ${ }^{1,2}$, Laurent A. Francis ${ }^{1}$ and \\ Denis Flandre ${ }^{1, *}$ \\ 1 ICTEAM Institute, Université catholique de Louvain (UCLouvain), Louvain-la-Neuve, 1348, Belgium; \\ rafael.puyoltroisi@uclouvain.be (R.P.); sylvain.petre@uclouvain.be (S.P.); yann.danlee@vocsens.com (Y.D.); \\ thomas.walewyns@vocsens.com (T.W.); laurent.francis@uclouvain.be (L.F.) \\ 2 VOCSens, Louvain-la-Neuve 1348, Belgium \\ * Correspondence: denis.flandre@uclouvain.be \\ + Presented at the 4th International Conference nanoFIS 2020-Functional Integrated nano Systems, Graz, \\ Austria, 2-4 November 2020.
}

Published: 16 December 2020

\begin{abstract}
Current sensing solutions must combine an ultra-low energy consumption trend with high reliability. The challenge lies on a fine setting of the detection threshold with the assurance of a sufficient sensitivity. In this article, the uncertainty introduced on gas sensing applications by the inherent sensor noise is studied. A 1/f model of the electronic noise in polypyrrole-based ammonia $\left(\mathrm{NH}_{3}\right)$ sensors is presented and used to estimate the intrinsic signal-to-noise ratio (SNR), giving an effective precision of 10.7 bits, i.e., down to $31.4 \mathrm{ppb}$ in terms of $\mathrm{NH}_{3}$ concentration. No significant improvement in SNR is achieved by increasing the bias voltage and hence the power consumption.
\end{abstract}

Keywords: gas sensing; sensor noise; polypyrrole sensor; ultra-low-power

\section{Introduction}

The measurement of a sensor returns an electrical signal, voltage or current related to a physical process that is to be estimated with maximum sensitivity. However, the signal can be corrupted by uncertainty due to sensor characteristics, functional material parameters, unwanted external signals, as well inherent electrical noise. In semiconductor-based thin films, flicker noise is frequently found in addition to thermal noise [1].

A great effort has been made to produce more selective and sensitive sensors but the overall signal-to-noise ratio (SNR) of the sensor, and hence the potential performance of the sensor interface, have been less studied. The detection threshold and the SNR are essential for a proper working of the system. In this article, the noise characteristics of polypyrrole (PPy)-based ammonia $\left(\mathrm{NH}_{3}\right)$ gas sensors are addressed. Firstly, the sensor is described, followed by noise measurements results. Thirdly, the uncertainty and SNR are discussed.

\section{Sensor Characterization}

The studied sensor is based on a PPy thin-film deposited over metallic interdigitated fingers made of gold on top of a 300-nm-thick $\mathrm{SiO}_{2}$ [2]. The size of the sensor is $1 \mathrm{~mm}$ by $1 \mathrm{~mm}$ and the interdigitated fingers cover almost all the area. The finger width is $4 \mu \mathrm{m}$ and the gap $2 \mu \mathrm{m}$.

The sensor, a resistor-like two-terminal device, was biased with a fixed voltage for each noise measurement using a E4727A Advanced Low-Frequency Noise Analyzer from Keysight. The current noise power spectral density (PSD) was obtained for different bias voltages, as shown in Figure 1a. 


\section{Results Analysis and Discussion}

The noise is dominated by flicker noise, no corner frequency being visible at least up to $100 \mathrm{kHz}$, and the theoretic thermal noise floor is $7.53 \times 10^{-24} \mathrm{~A}^{2} / \mathrm{Hz}$, which translates into a corner frequency in the order of $\mathrm{MHz}$. It should be noted that gas concentration variations correspond, in general, to slow processes in the order of seconds or fractions of seconds; therefore, a pure flicker noise model is sufficient to estimate the total noise, since higher frequency noise is to be filtered. The flicker noise coefficients were extracted for every curve by integration of the PSD.

(a)

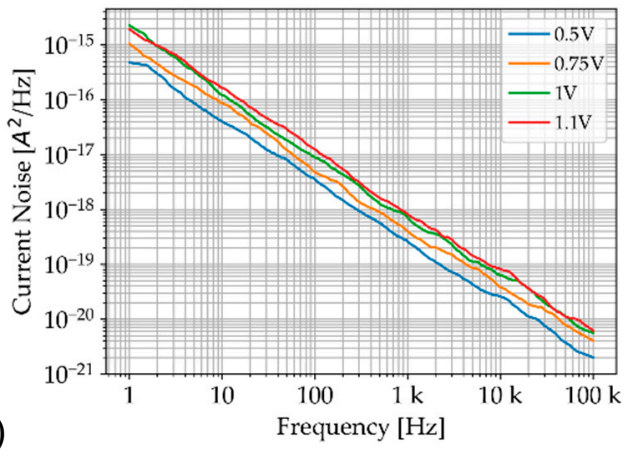

(b)

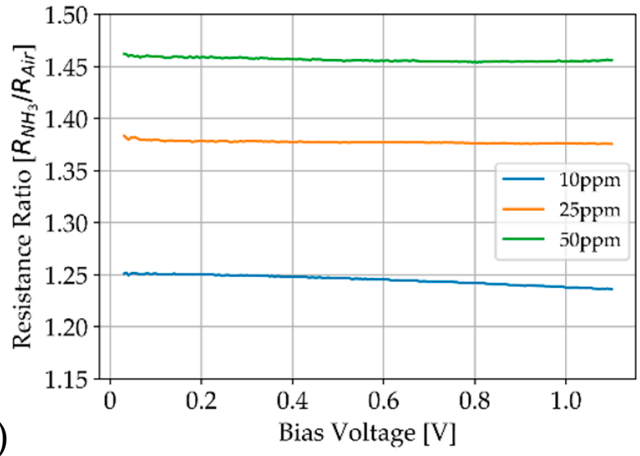

Figure 1. (a) Voltage noise power spectral density (PSD) for different bias voltages in air at $22{ }^{\circ} \mathrm{C}, 35 \%$ $\mathrm{RH}$. (b) Sensor resistance variation with bias voltage for the different ammonia gas concentrations referenced to clean air.

The sensitivity of the sensor has been measured for a wide range of bias voltages and ammonia concentrations and shown to be mostly unaffected by the bias voltage below $1 \mathrm{~V}$; see Figure $1 \mathrm{~b}$. It is non-linear, ranging from $2.5 \% / \mathrm{ppm}$ at $10 \mathrm{ppm}$ down to $0.92 \% / \mathrm{ppm}$ at $50 \mathrm{ppm}$. The sensor current and integrated RMS noise are listed in Table 1, as well as the SNR and the effective number of bits (ENOB). The sensor resistance is weakly dependent on the bias voltage. The total RMS noise appears sufficiently low for sub-ppm resolution in our measurement range, with precision as low as $31.4 \mathrm{ppb}$ $\mathrm{NH}_{3}($ at $10 \mathrm{ppm}$ ) and detection limit far below $100 \mathrm{ppb}$.

Table 1. Signal, Noise, SNR and ENOB values for each bias voltage in air.

\begin{tabular}{ccccc}
\hline Bias Voltage (V) & Sensor Current $(\boldsymbol{\mu A})$ & Total RMS Noise (nA) & SNR (dB) & ENOB (bits) \\
\hline 0.5 & 201.6 & 98.65 & 66.2 & 10.7 \\
0.75 & 330.8 & 160.0 & 66.3 & 10.7 \\
1 & 475.3 & 179.8 & 68.5 & 11.1 \\
1.1 & 543.8 & 209.7 & 68.3 & 11.1 \\
\hline
\end{tabular}

An important result, obtained from Table 1, is that after increasing the current by a factor of 2.7 and the power by 6, the gain in SNR is marginal since the flicker noise power also shows a dependence on the square of the current. Although from a circuit analysis point of view the sensor can be modeled as a resistor, this is not true for the noise analysis. This result proves very useful in ultra-low-power sensing applications, such as Internet of Things nodes, where a trade-off between battery life and sensing precision must be dealt with. In particular, designing a sensor interface with a precision better than 10 bits would not prove valuable.

\section{Conclusions}

An analysis of the effects of intrinsic electrical noise on sensitivity for $\mathrm{PPy}_{\mathrm{NH}}$ sensors was presented. Flicker noise was shown to dominate and to yield a power spectral density dependent on bias condition. This does not allow for a significant gain in SNR by increasing the bias voltage. Though, its knowledge is a key point for reliable measurements towards the development of ultra- 
low-power portable device and optimal front-end analog interface design. This characterization study next allows for optimizing the power management while still ensuring a high accuracy at the system level.

\section{References}

1. Makoviychuk, M.I.; Chapkevich, A.L.; Vinokurov, V.A. Flicker-noise gas sensor. Biomed. Eng. 2009, 43, 109-113.

2. Marchand, N.; Walewyns, T.; Lahem, D.; Debliquy, M.; Francis, L.A. Ultra-low-power chemiresistive microsensor array in a back-end CMOS process towards selective volatile compounds detection and IoT applications. In Proceedings of the ISOCS/IEEE International Symposium on Olfaction and Electronic Nose (ISOEN), Montreal, QC, Canada, 28-31 May 2017.

Publisher's Note: MDPI stays neutral with regard to jurisdictional claims in published maps and institutional affiliations.

(C) 2020 by the authors. Licensee MDPI, Basel, Switzerland. This article is an open access article distributed under the terms and conditions of the Creative Commons Attribution (CC BY) license (http://creativecommons.org/licenses/by/4.0/). 